\title{
Rescue of rhesus macaques from the lethality of aerosolized ricin toxin
}

\author{
Chad J. Roy, ${ }^{1}$ Dylan J. Ehrbar, ${ }^{2}$ Natasha Bohorova, ${ }^{3}$ Ognian Bohorov, ${ }^{3}$ Do Kim, ${ }^{3}$ Michael Pauly, ${ }^{3}$ \\ Kevin Whaley, ${ }^{3}$ Yinghui Rong, ${ }^{2}$ Fernando J. Torres-Velez, ${ }^{2}$ Ellen S. Vitetta, ${ }^{4}$ Peter J. Didier, ${ }^{1}$ \\ Lara Doyle-Meyers, ${ }^{1}$ Larry Zeitlin, ${ }^{3}$ and Nicholas J. Mantis ${ }^{2}$ \\ 'Tulane National Primate Research Center (TNPRC), Covington, Louisiana, USA. ²Division of Infectious Disease, \\ Wadsworth Center, New York State Department of Health, Albany, New York, USA. ${ }^{3}$ Mapp Biopharmaceutical Inc., \\ San Diego, California, USA. ${ }^{4}$ Departments of Immunology and Microbiology, University of Texas Southwestern Medical \\ Center, Dallas, Texas, USA.
}

Ricin toxin (RT) ranks at the top of the list of bioweapons of concern to civilian and military personnel alike, due to its high potential for morbidity and mortality after inhalation. In nonhuman primates, aerosolized ricin triggers severe acute respiratory distress characterized by perivascular and alveolar edema, neutrophilic infiltration, and severe necrotizing bronchiolitis and alveolitis. There are currently no approved countermeasures for ricin intoxication. Here, we report the therapeutic potential of a humanized mAb against an immunodominant epitope on ricin's enzymatic $A$ chain (RTA). Rhesus macaques that received i.v. huPB10 4 hours after a lethal dose of ricin aerosol exposure survived toxin challenge, whereas control animals succumbed to ricin intoxication within $\mathbf{3 0}$ hours. Antibody intervention at 12 hours resulted in the survival of 1 of 5 monkeys. Changes in proinflammatory cytokine, chemokine, and growth factor profiles in bronchial alveolar lavage fluids before and after toxin challenge successfully clustered animals by treatment group and survival, indicating a relationship between local tissue damage and experimental outcome. This study represents the first demonstration, to our knowledge, in nonhuman primates that the lethal effects of inhalational ricin exposure can be negated by a drug candidate, and it opens up a path forward for product development.

Conflict of interest: $\mathrm{NB}, \mathrm{OB}, \mathrm{DK}$, and MP are Mapp Biopharmaceutical employees and shareholders. KW and LZ are employees, shareholders, and coowners of Mapp Biopharmaceutical.

License: Copyright 2019, American Society for Clinical Investigation.

Submitted: September 7, 2018 Accepted: November 27, 2018 Published: January 10, 2019

Reference information: JCI Insight. 2019;4(1):e124771. https:// doi.org/10.1172/jci.insight.124771.

\section{Introduction}

Ricin toxin (RT) is considered a high priority biothreat agent by the Centers for Disease Control and Prevention (CDC), the US Department of Defense (DOD), and the North Atlantic Treaty Organization (NATO) due to its accessibility, stability, and high toxicity, especially by the aerosol route $(1,2)$. In nonhuman primates (NHPs), inhalation of RT elicits the clinical equivalent of acute respiratory distress syndrome (ARDS), characterized by widespread apoptosis of alveolar macrophages, intraalveolar edema, neutrophilic infiltration, accumulation of proinflammatory cytokines, and fibrinous exudate (3-5). Similar effects are observed in mice, rats, and swine (6-11). RT is derived from castor beans (Ricinus communis) and is a $65-\mathrm{kDa}$ heterodimeric glycoprotein consisting of 2 subunits, RTA and RTB, joined via a single disulfide bond. RTB binds to glycoproteins and glycolipids on mammalian cells and facilitates the retrograde transport of RT to the ER. In the ER, RTA is liberated from RTB and retrotranslocated into the cytoplasm via the Sec61 complex (12). RTA is an RNA N-glycosidase that depurinates a conserved adenosine residue within the sarcin/ricin loop of $28 \mathrm{~S}$ rRNA, resulting in the inhibition of protein synthesis $(13,14)$ and the activation of apoptosis (15). Alveolar macrophages are particularly sensitive to the cytotoxic effects and secrete an array of proinflammatory cytokines before undergoing apoptosis $(8,9,16)$.

In this report, we investigated the potential of a humanized mAb huPB10 to serve as a therapeutic in an established Rhesus macaque model of RT inhalation $(4,17)$. PB10 was first described as a murine $\mathrm{mAb}$ with potent toxin-neutralizing activity in vitro and in vivo (18). PB10 recognizes an immunodominant $\alpha$-helix situated near RTA's active site, although the actual mechanism by which it neutralizes ricin remains unknown $(19,20)$. Chimeric and fully humanized versions of PB10 (cPB10 and huPB10, respectively) have been shown to passively protect mice against $10 \times \mathrm{LD}_{50}$ of $\mathrm{RT}$ administered by injection or inhalation $(21,22)$. PB10 has also been demonstrated to have therapeutic value, as it can rescue 
mice from ricin if administered within 4-6 hours after toxin exposure (22). Based on these and other preliminary findings, we sought to examine the therapeutic potential of huPB10 in a well-established NHP model of ricin aerosol challenge (17).

\section{Results}

Experimental design. A total of 12 rhesus macaques ( $7 \mathrm{~kg}$; range $3.8-10.2 \mathrm{~kg}$ ) bred at TNPRC were randomly assigned to 3 experimental groups and then challenged at time designated zero with RT by small-particle aerosol at a target dose of $18 \mu \mathrm{g} / \mathrm{kg}$ or the equivalent of $\sim 3 \times \mathrm{LD}_{50}$ (Table 1 and Figure 1). The control group $(n=2)$ received i.v. administration of saline 4 hours after ricin challenge. The second group of animals $(n=5)$ received huPB10 $(10 \mathrm{mg} / \mathrm{kg})$ i.v. at 4 hours after ricin challenge, while a third group $(n=5)$ received huPB10 $(10 \mathrm{mg} / \mathrm{kg})$ at 12 hours (Table 1$)$. The macaques were subjected to whole body plethysmography and radiotelemetry over the course of the study. Sera and bronchial alveolar lavage fluids (BALF) were collected from the animals 7 days before and 24 hours after RT exposure, although - for technical reasons - BALF were only obtained from 1 of the control animals after ricin challenge. Animals that survived to day 14 were euthanized and subjected to complete necropsy and histopathological analysis.

Clinical outcomes of control and huPB10 animals following RT exposure. The control animals succumbed to RT toxicosis by 30 hours (Table 1 and Figure 1). The clinical progression of RT-induced intoxication was similar to that observed in previous studies (4). Approximately 12-16 hours after exposure, animals displayed reduced activity and fever (Figure 2). Clinical examination conducted 24 hours after exposure revealed respiratory complications, including bilateral congestion and crackles, with dyspnea and tachypnea. Arterial oxygen ranged from $75 \%-85 \%$, as compared with normal saturation levels of $96 \%-99 \%$. Prominent tachycardia accompanied low blood oxygen readings. The clinical state of the animals continued to decline over the next several hours, manifested by a marked drop in normal activity and the appearance of cyanotic mucous membranes. The animals that received huPB10 12 hours after ricin challenge fared only marginally better than the control animals in that just 1 of the 5 macaques survived to day 14; the other 4 animals followed a clinical course similar to the control animals and expired between 44 and 72 hours after ricin challenge (Table 1 and Figure 1). In samples collected at 24 hours after ricin challenge, the average concentration of huPB10 in the 12-hour treatment groups was $203 \mu \mathrm{g} / \mathrm{ml}$ in serum and $17.3 \mu \mathrm{g} / \mathrm{ml}$ in BALF (Table 1).

In stark contrast, all 5 of the Rhesus macaques that received huPB10 at 4 hours survived ricin exposure $(P<0.01$, compared with controls) and remained otherwise normal for the duration of the 2 -week observation period (Table 1 and Figure 1). In the physical examination conducted 24 hours after exposure, the 5 macaques presented with only minimal signs of distress (e.g., mild dyspnea, minimal tachypnea, and increased lung sounds); no such symptoms were evident upon a second physical examination conducted on day 7. In samples collected at 24 hours after ricin challenge, the average concentration of huPB10 was 182 $\mu \mathrm{g} / \mathrm{ml}$ in serum and $3 \mu \mathrm{g} / \mathrm{ml}$ in BALF (Table 1 ).

Pathology associated with RT exposure. Gross examination of the lungs from control animals revealed coalescing hemorrhage with frothy exudate marked by fibrin in lung parenchyma (Supplemental Figure 1; supplemental material available online with this article; https://doi.org/10.1172/jci.insight.124771DS1). There was evidence of fibrinosuppurative bronchointerstitial pneumonia with pulmonary edema and bronchial epithelial necrosis, with severe fibrinosuppurative lymphadenitis in the bronchial lymph nodes. The wet weights of the lungs from the control animals collected upon necropsy were $>150 \mathrm{~g}$ in weight, compared with $\sim 30-40 \mathrm{~g}$ in normal animals of the same body weight, based on TNPRC historical data. Histologically, the lungs of the control animals had hallmarks of RT-induced injury: marked edema, corresponding hemorrhage, and numerous polymorphonuclear neutrophils (PMN) and eosinophils (Figure 2).

The pathological outcome of the 4 animals that succumbed to RT intoxication in the group that the received huPB10 at 12 hours resembled the control animals. There was extensive pulmonary congestion, edema, inflammation characterized by the infiltration of numerous PMN, and punctate hemorrhage evident. Gross lung weights were similar to RT-challenged control animals ( $>140 \mathrm{~g}$ ), and there were clear signs of hemorrhage, though not as severe as the controls, suggesting that huPB10 intervention did alter the course of the inflammatory response.

Lung tissues collected at the time of necropsy on day 14 after challenge from the 5 animals that received huPB10 at 4 hours, along with the single survivor (DR61) in the group that received huPB10 at 12 hours, were considered grossly normal. However, closer examination revealed evidence of chronic inflammation 
Table 1. Experimental groups and outcome of ricin challenge

\begin{tabular}{|c|c|c|c|c|c|c|c|c|}
\hline \multirow[b]{2}{*}{ Group } & \multirow[b]{2}{*}{ ID } & \multirow[b]{2}{*}{ kg } & \multicolumn{6}{|c|}{ huPB10 $(\mu \mathrm{g} / \mathrm{ml})^{c}$} \\
\hline & & & $\mathbf{M} / F^{A}$ & $\mathbf{R T}^{\mathrm{B}}$ & serum & BAL & TTD $^{\mathrm{D}}$ & live/total \\
\hline \multirow[t]{2}{*}{ Control } & $\mathrm{DH} 27$ & 9.7 & $M$ & 14 & - & - & $28 \mathrm{~h}$ & \\
\hline & JP56 & 8.4 & $M$ & 26 & - & - & $30 \mathrm{~h}$ & $0 / 2$ \\
\hline \multirow[t]{5}{*}{ +4 h huPB10 } & LJ80 & 4.4 & $M$ & 31 & 157 & 2.8 & + & \\
\hline & LB98 & 4.9 & $M$ & 29 & 177 & 2.7 & + & \\
\hline & EM61 & 9.5 & $M$ & 31 & 207 & 2.5 & + & \\
\hline & IC70 & 5.9 & $\mathrm{~F}$ & 26 & 225 & 3.6 & + & \\
\hline & IL-40 & 6.1 & $\mathrm{~F}$ & 33 & 147 & 3.2 & + & $5 / 5$ \\
\hline \multirow[t]{5}{*}{ +12 h huPB10 } & JM39 & 9.4 & $M$ & 22 & 205 & 42.8 & $72 \mathrm{~h}$ & \\
\hline & DR61 & 10.2 & $\mathrm{~F}$ & 24 & 265 & 3.9 & + & \\
\hline & KР48 & 5.8 & $M$ & 30 & 203 & 25.7 & $48 \mathrm{~h}$ & \\
\hline & KR49 & 5.7 & $M$ & 29 & 151 & 3.1 & $49 \mathrm{~h}$ & \\
\hline & LB26 & 3.8 & $M$ & 29 & 191 & 11.3 & $44 \mathrm{~h}$ & $1 / 5$ \\
\hline
\end{tabular}

${ }^{A}$ Sex of animal; Bricin toxin (RT) dose $(\mu \mathrm{g} / \mathrm{kg}$ ) received per animal; ChuPB10 in serum and BAL from samples collected at 24 hours after challenge. The average in serum was $182.6 \mu \mathrm{g} / \mathrm{ml}$ and $203 \mu \mathrm{g} / \mathrm{ml}$ for the +4 - and +12 -hour groups, respectively. In the BAL, there was $3.04 \mathrm{~g} / \mathrm{ml}$ and $17.3 \mu \mathrm{g} / \mathrm{ml}$, respectively. Times of huPB10 administration are approximate ( 30 minutes of point estimate); the logistics of performing slow i.v. infusion of huPB10 in monkeys initiated 4 hours from each of the individual ricin aerosol challenge events and was performed in sequence. ${ }^{D}$ Time to death (TTD). The + symbol indicates survival for 14-day duration of study.

and a distinctive fibrosis proximal to the respiratory bronchioles that was reminiscent of animals that have been exposed to sublethal doses of RT (Figure 3) (4, 23). Thus, toxin-induced tissue damage was greatly attenuated, but not completely abrogated, in Rhesus macaques that survived aerosolized ricin exposure as a result of huPB10 intervention.

Profiling of serum and BAL inflammatory markers following RT exposure. To assess the impact of huPB10 intervention on local and systemic inflammatory responses following RT exposure, we subjected sera and BALF collected before (day -7) and 24 hours after RT challenge to analysis with a 29-plex cytokine, chemokine, and growth factor Luminex array. The repeated measures design of our analysis (i.e., comparing samples collected before versus after RT challenge) allowed us to determine significant changes for many of the analytes examined, despite having only 2 animals in the control group.

With respect to inflammatory markers in serum, a total of 12 analytes were significantly changed following RT exposure in the 2 control animals (Figure 4 and Supplemental Table 2). Most notable were IL-6 and IL-1RA, which increased by approximately 600 - and $~ 250$-fold, respectively. VEGF was elevated 11-fold, GCSF increased by approximately 18-fold, and MCP1 increased by approximately 12-fold. Other more modest increases included MIF ( $\sim$-fold) and GMCSF ( 4-fold), as well as IL-5 and IL-2, which each increased $\sim 3$-fold. Finally, levels of IL- 8 were significantly reduced in the 2 control serum samples following RT exposure.

In animals that received huPB10 at 12 hours, a total of 6 cytokines/chemokines/growth factors were changed relative to prechallenge levels, including a downregulation of IL-8, although the magnitude of these changes was less than that in observed in the control animals. A dampening of the inflammatory response due to huPB10 intervention was even more pronounced in the group of animals that received antibody treatment at 4 hours, as evidenced by only a $\sim 4$-fold increase in IL- 6 . IL- 8 was the only chemokine to be significantly downregulated in all groups following ricin challenge.

The impact of huPB10 intervention was much more apparent in BALF than in sera (Figure 5 and Supplemental Table 2). In the group of animals that received huPB10 at 12 hours, there were 25 analytes that were significantly elevated compared with prechallenge levels. IL- 6 was again the most pronounced (increased $\sim 167$-fold), followed by a 5 - to 20 -fold increases in MCP-1, eotaxin, IL-1RA, and MDC. IL-1B, GCSF, and IL-5 increased between 3- to 4-fold. In the group of animals that received huPB10 at 4 hours, a total of 21 inflammatory markers were significantly elevated following RT challenge, as compared with prechallenge levels, with IL-6 being the most pronounced (elevated 27-fold). MCP-1 was 

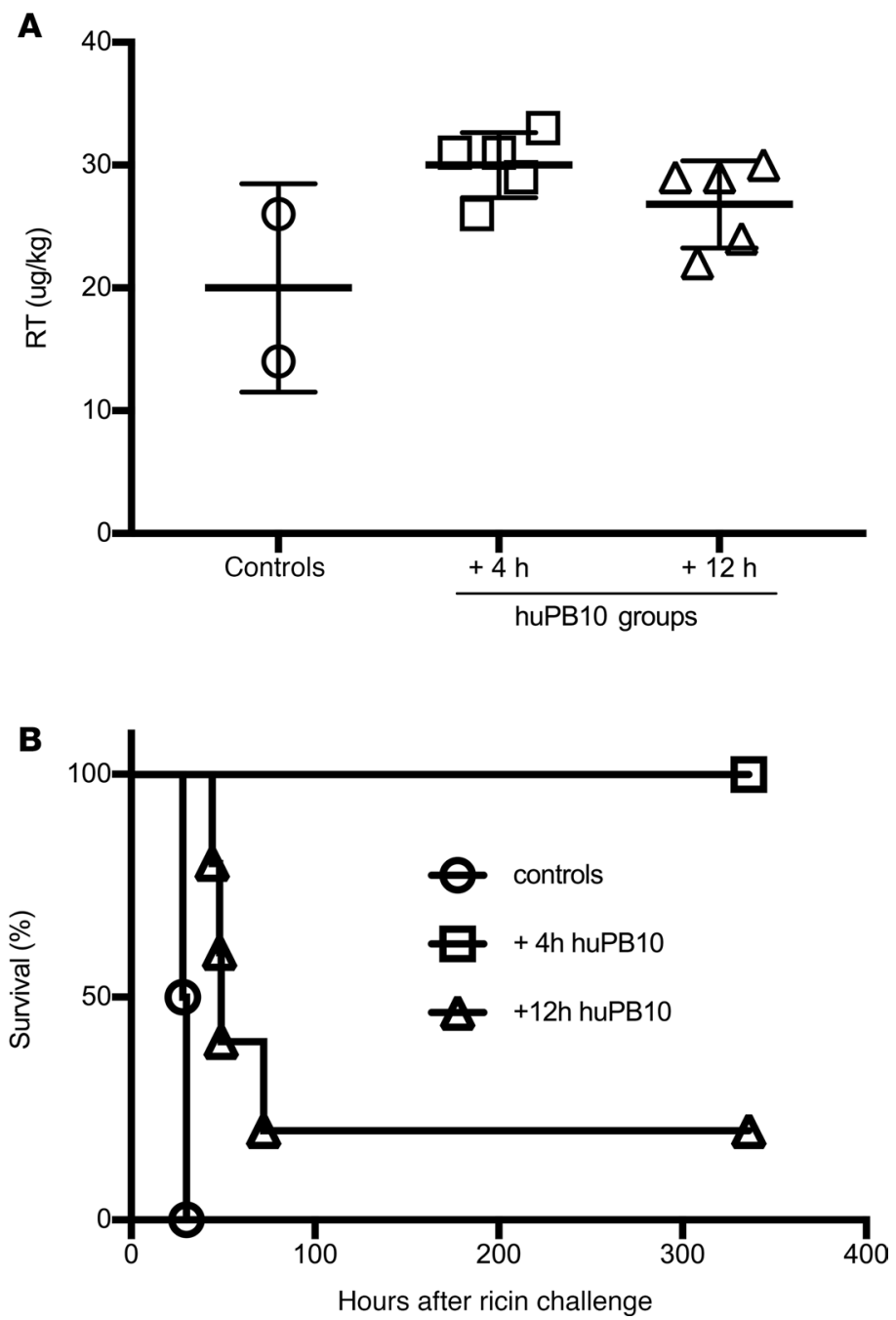

Figure 1. I.v. huPB10 treatment rescues Rhesus macaques exposed to lethal dose of aerosolized RT. (A) Groups of Rhesus macaques were exposed to aerosolized RT. Shown are the individual RT doses ( $\mu \mathrm{g} / \mathrm{kg}$ inhaled) by treatment group. Bars represent the group mean \pm SD. The target dose $(18 \mu \mathrm{g} / \mathrm{kg})$ is represented by a segmented line. (B) Rhesus macaque survival presented as Kaplan-Meier curve. The control animals $(n=2)$ succumbed to ricin intoxication by day 2. The animals $(n=5)$ that received huPB10 at 4 hours after challenge survived for the duration of the study. Only 1 animal in the group $(n=5)$ that received huPB10 at 12 hours after ricin challenge survived.

also increased ( 9-fold), as were MDC, eotaxin, and ITAC (3-4-fold). IL-1RA, IL-1B, MIP1A, MIF, and IL-8 each rose $\sim 2$-fold. Between the 4-hour and 12-hour huPB10 intervention groups, there were 7 analytes that were significantly different (i.e., lower in the 4-hour groups compared with the 12-hour group), including IL-6, IL-5, GMCSF, and IL-2. Thus, the breadth and the magnitude of the inflammatory response in the lung was significantly reduced in the animals that received huPB10 as 4 hours, compared with the group that received antibody at 12 hours.

We next subjected the serum samples and BALF to principle component analysis (PCA) and hierarchical clustering to assess whether there exists a relationship between changes in systemic and/or local inflammatory markers and experimental outcome (i.e., survival). PCA of serum samples (fold change values before versus after challenge) from all 12 monkeys did not yield any meaningful relationships with experimental group or survival (Figure 6), signifying that serum inflammatory responses are not indicative of experimental outcome. Hierarchical clustering of macaques based on fold changes of serum analytes also failed to group the animals by either survival status or group membership (Supplemental Figure 3).

However, PCA of BALF successfully clustered animals by both group and survival status, with IL-2, IL-5, GMCSF, MIF, IP10, IL-12, HGF, RANTES, IL-6, and TNFA being the primary contributors to principle components 1 and 2 (Figure 6). Hierarchical clustering of monkeys based on fold change values of BAL 
A

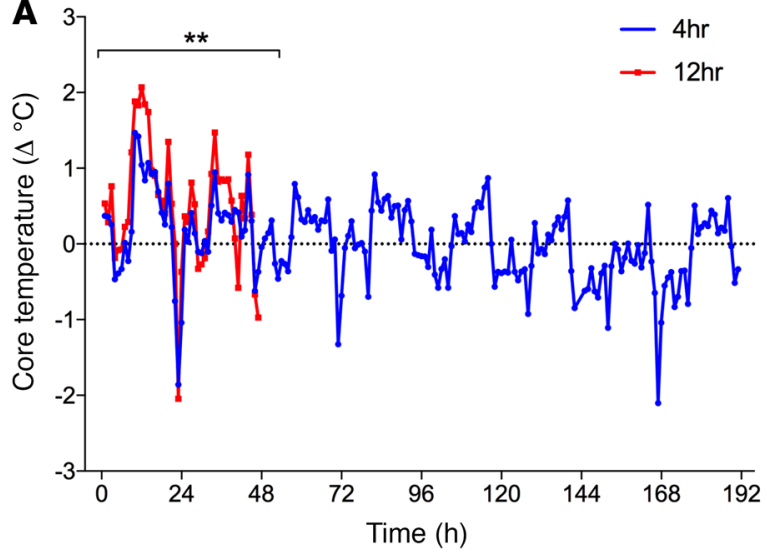

B
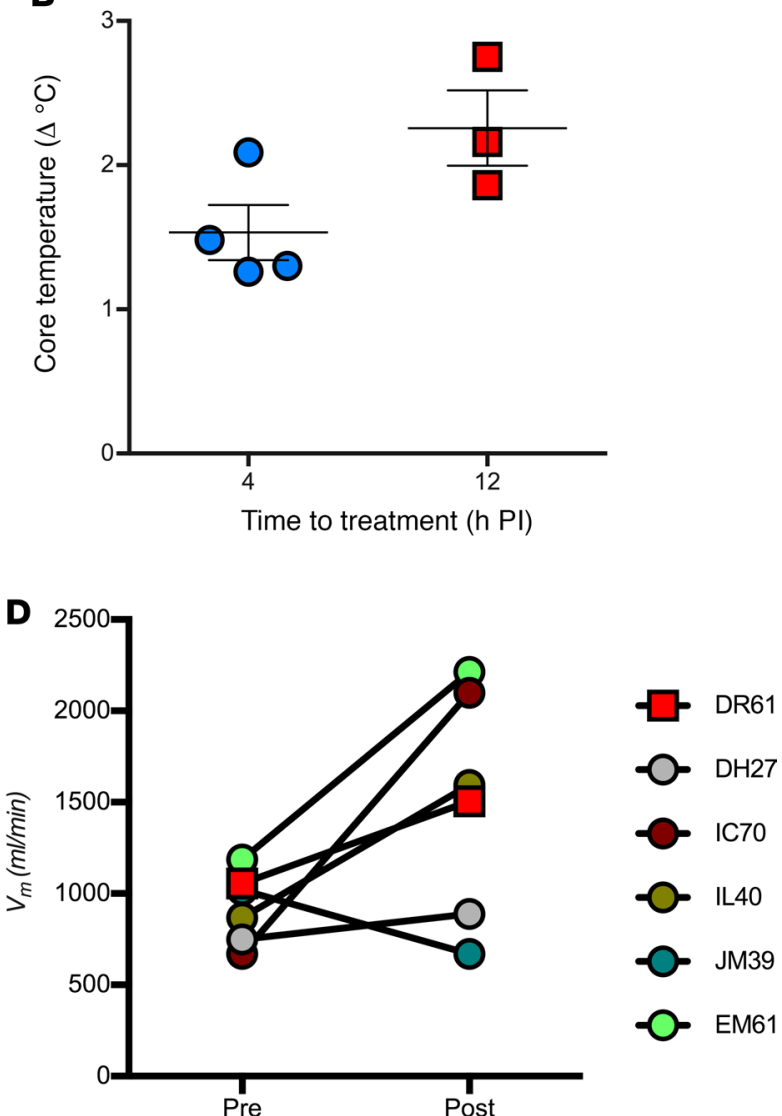

Figure 2. Physiological response of Rhesus macaques to RT exposure. (A) Radiotelemetry of core temperature of Rhesus macaques treated with huPB10 either at 4 hours or 12 hours after exposure to aerosolized RT. Continuous monitoring showed significant differences $(P<0.005)$ between the initiation and tempo of pyrexia that was dependent on the time to treat. (B) Fever intensity showed differences between the relative change (increase) based upon time to treat. Respiratory function measured by whole body plethysmography. Respiratory function was measured via head-out conductance plethysmography prior to and at timed intervals after challenge. (C) Significant changes in post-exposure group tidal volume $\left(V_{t}\right)$ was observed among animals rescued from ricin intoxication ( $P$ $<0.05$ by 2 -way ANOVA). (D) However, compensatory changes in frequency resulted in minimal observable changes in minute volume ( $\left.V_{m}\right)$, with some animals producing increased minute volumes +24 hours after exposure to ricin $(P=0.08)$. Animal DR61 (square symbols) was the sole animal that survived RT exposure within the group that received huPB10 at 12 hours.

cytokines also indicated that macaques tended to cluster separately based on survival and group membership (Supplemental Figure 4). Thus, survival outcome of Rhesus macaques following RT exposure is more closely related to the local inflammatory responses in the lung than in the disseminated responses measured in serum.

\section{Discussion}

This study constitutes a significant advance in a longstanding effort to develop effective medical countermeasures against a persistent biothreat agent, as it is the first demonstration to our knowledge that a drug candidate can rescue NHPs from the lethal effects of aerosolized RT exposure $(2,24,25)$. The success of this study is the result of 2 convergent advances in the ricin research community: (a) the availability of a well-characterized humanized $\mathrm{mAb}$ with demonstrated in vivo activity against $\mathrm{RT}$ in mice and $(\mathrm{b})$ an aerosolized RT challenge model in Rhesus macaques with clearly defined clinical and pathological outcomes $(4,17,23,26)$. PB10 is a high-affinity $\mathrm{mAb}$ that recognizes an immunodominant toxin-neutralizing epitope on RTA, as demonstrated by peptide mapping and hydrogen-deuterium exchange/mass spectrometry (18-20). A humanized version of PB10 was successfully expressed in a scalable, Nicotiana-based platform and shown to passively protect mice against RT challenge by different routes of exposure (21, 22, 27). Moreover, we reported a threshold serum concentration of $\sim 18 \mu \mathrm{g} / \mathrm{ml}$ huPB10 as being required to protect mice against $10 \times \mathrm{LD}_{50}$ i.n. RT challenge (22).

By the same token, the Rhesus macaque has been successfully developed as a model to study clinical, pathological, and inflammatory responses in the lung and serum associated with lethal and sublethal ricin aerosol exposures $(4,23)$. Most recently, the model was used to demonstrate the efficacy of a recombinant 

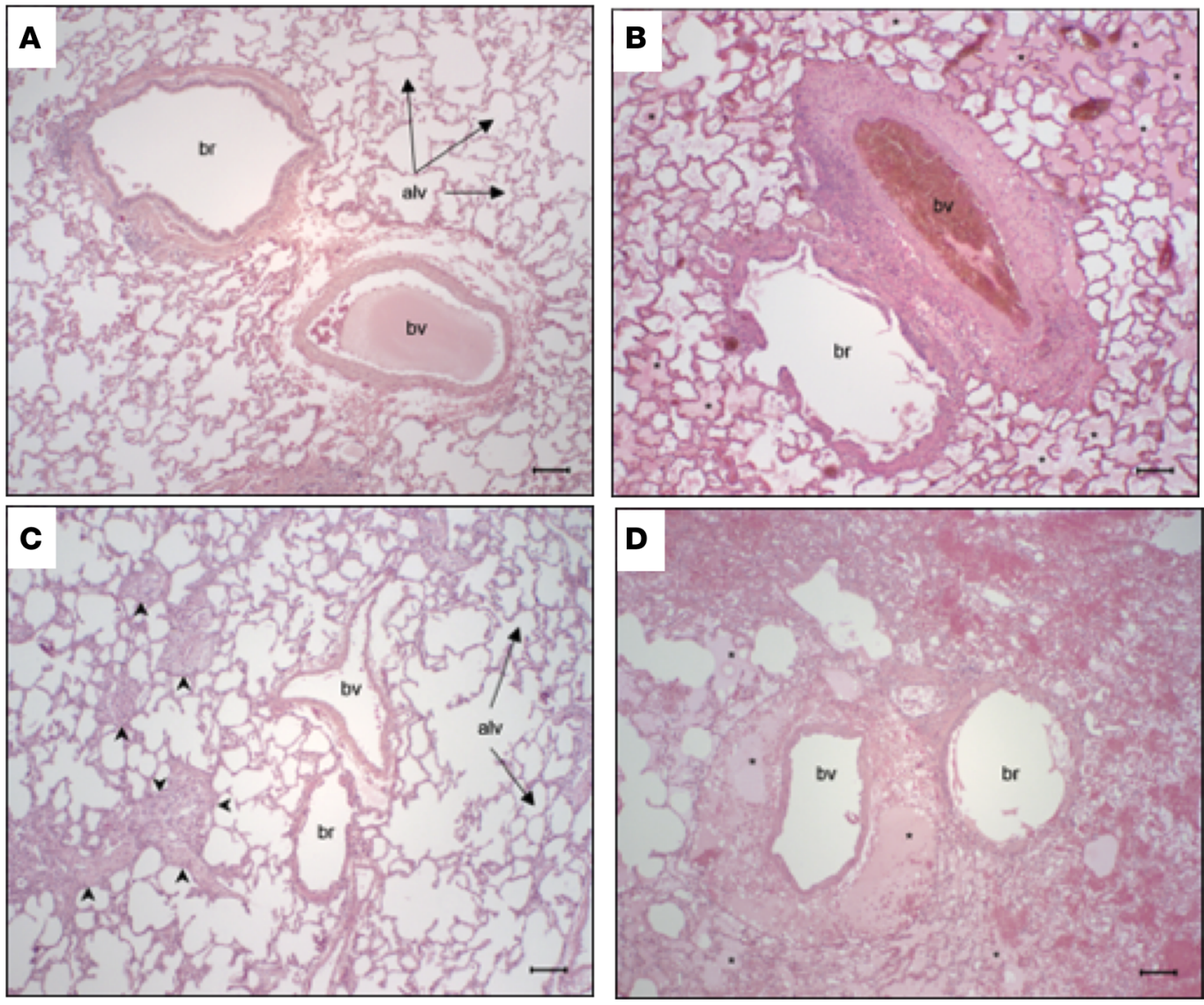

Figure 3. Histopathology associated with RT exposure and huPB10 intervention. H\&E-stained tissue sections of lung tissues collected from (A) healthy control animals, (B) RT-treated animals, and RT-treated animals that received huPB10 at (C) 4 hours or (D) 12 hours. Tissues in $\mathbf{B}$ and $\mathbf{D}$ were collected during necropsy from animals that succumbed to ricin intoxication, whereas the tissue in $\mathbf{C}$ was collected from an animal that survived RT exposure but was euthanized upon completion of the study on day 14. Tissues from (B) RT-treated and the (D) huBP10 intervention at 12 hours demonstrated severe neutrophilic infiltration of bronchioles (br), space around blood vessels (bv), and interstitium, as compared with healthy controls shown in A. Alveolar spaces (alv; arrows) of RT and 12 hour-treated animals were expanded by edema (asterisks) and hemorrhage. (C) Animals treated with huPB10 at 4 hours demonstrated multifocal areas of fibrosis and mild eosinophilic and neutrophilic infiltration (arrowheads; see Supplemental Figure 2). Original magnification 10x. Scale bar: $100 \mu \mathrm{m}$.

RTA subunit vaccine (RiVax) administered i.m. in conferring immunity against $3 \times \mathrm{LD}_{50}$ inhalation toxin challenge (17). In that study, a total of 6 sham vaccinated animals were challenged with RT, thereby providing a robust database of clinical and pathological metrics associated with toxin exposure (4). Survival of the vaccinated animals in the face of aerosolized RT challenge was associated with RTA-specific serum IgG antibodies, including a proportion directed against PB10's epitope. The geometric mean concentration of anti-RTA IgG in serum at the time of challenge was $\sim 360 \mu \mathrm{g} / \mathrm{ml}$, which is comparable with the levels of huPB10 that were achieved by i.v. delivery (Table 1). Unfortunately, for reasons related to cost and experimental logistics, we were unable to perform huPB10 dose titration studies in the Rhesus macaques.

When considering the relatively short therapeutic window associated with huPB10, it should be noted that the $3 \times \mathrm{LD}_{50}$ challenge dose used here is, by all accounts, extremely severe. For example, the 2 control animals, which received 14 and $28 \mu \mathrm{g} / \mathrm{kg} \mathrm{RT}$, expired at 28 and 30 hours, respectively. This time frame is essentially identical to the mean time to death reported in the ricin vaccine study, even though the actual doses of RT vary due to inherent differences in individual respiratory function $(4,17)$. As a point of reference, a target dose of $18 \mu \mathrm{g} / \mathrm{kg}$ RT is roughly equivalent to deposition of 100-200 $\mu \mathrm{g}$ toxin in the lungs. While the exact distribution and pharmacokinetics of uptake of RT in the lung following inhalation has been difficult to discern, it is clear that the toxin acts locally in a time-, dose-, and cell type-dependent manner, with alveolar macrophages being an early and particularly sensitive 


\begin{tabular}{|c|c|c|c|c|c|c|c|c|c|c|c|c|}
\hline & \multicolumn{2}{|c|}{ Control } & \multicolumn{5}{|c|}{$4 \mathrm{hr}$ intervention } & \multicolumn{5}{|c|}{$12 \mathrm{hr}$ intervention } \\
\hline EGF & 1.53 & 0.83 & -0.17 & 0.73 & 0.28 & -0.83 & 0.64 & 0.27 & 0.64 & -0.67 & 0.79 & 1.42 \\
\hline Eotaxin & 0.62 & 0.91 & -0.01 & 0.48 & 0.31 & 0.28 & 0.40 & -0.29 & 0.01 & -0.05 & -0.17 & 0.61 \\
\hline FGF basic & 0.38 & 1.49 & 0 & 0.33 & -0.27 & -0.32 & 0.40 & 0.25 & 0.28 & -0.35 & 0.29 & 0.73 \\
\hline GCSF & 2.58 & 4.92 & -0.07 & 0.05 & -0.93 & -0.22 & -0.33 & -0.14 & -0.21 & -0.33 & -0.10 & 0.21 \\
\hline GMCSF & 1.58 & 2.16 & -0.21 & -0.05 & 0.08 & -0.07 & 0.07 & -0.03 & 0.04 & -0.18 & -0.07 & 0.26 \\
\hline HGF & 0.33 & 0.68 & -0.24 & -0.10 & -1.07 & -0.22 & 0.04 & -0.17 & -0.16 & -0.04 & -0.15 & 0.23 \\
\hline IFNG & 0.53 & 0.74 & -0.20 & 0.05 & -1.15 & -0.22 & 0 & -0.13 & -0.21 & 0 & -0.24 & 0.57 \\
\hline IL1B & 0.99 & 1.56 & -0.15 & -0.01 & -0.33 & -0.06 & 0.15 & -0.06 & -0.03 & -0.07 & -0.38 & 0.44 \\
\hline IL1RA & 7.17 & 8.47 & 0.09 & 0.04 & 0.12 & -0.47 & 1.73 & 1.68 & 0.84 & 1.55 & 1.35 & 3.74 \\
\hline IL2 & 1.04 & 1.64 & -0.08 & -0.10 & 0.10 & 0 & 0.07 & -0.07 & 0.07 & -0.03 & -0.21 & 0.13 \\
\hline IL4 & 0.23 & 0.35 & -0.15 & -0.08 & -0.58 & -0.12 & -0.11 & 0 & -0.07 & 0.08 & -0.14 & -0.07 \\
\hline IL5 & 1.04 & 1.95 & -0.21 & 0.26 & -0.97 & -0.39 & 0.18 & -0.06 & 0.15 & -0.20 & -0.38 & 0.06 \\
\hline IL6 & 8.65 & 9.64 & 1.41 & 1.94 & 2.23 & 2.14 & 2.61 & 2.30 & 0.64 & 2.49 & 2.24 & 4.18 \\
\hline IL8 & -0.85 & -1.52 & -1.74 & -1.87 & -1.83 & -2.01 & -0.84 & -2.28 & -2.80 & -1.78 & -3.56 & -1.90 \\
\hline IL10 & 0.60 & 0.85 & -0.06 & -0.03 & -0.77 & -0.03 & 0.06 & -0.01 & 0.10 & 0.05 & 0 & 0.12 \\
\hline IL12 & -0.44 & 0.05 & -0.13 & -0.27 & -0.82 & -0.43 & 0.07 & -0.32 & -0.25 & -0.10 & -0.22 & -0.55 \\
\hline IL15 & 0.57 & 0.96 & -0.18 & 0.05 & -0.61 & -0.16 & 0.27 & -0.08 & 0.02 & -0.45 & -0.11 & 0.21 \\
\hline IL17 & 0.05 & -0.08 & -0.10 & -0.12 & -0.23 & -0.40 & 0.04 & -0.21 & -0.18 & -0.42 & -0.18 & 0.14 \\
\hline ITAC & -0.32 & -0.17 & -0.57 & -0.74 & 0.50 & -0.56 & 0.50 & -0.19 & -0.81 & -0.71 & -0.36 & 0.18 \\
\hline MCP1 & 3.05 & 4.02 & -0.16 & 0.73 & 0.72 & 0.28 & 1.68 & -0.22 & 0.30 & 0.26 & -0.31 & 1.41 \\
\hline MDC & -0.25 & 0.19 & -0.16 & -0.28 & -0.04 & 0.17 & 0.33 & 0 & 0.23 & 0.08 & -0.42 & -0.36 \\
\hline MIF & 2.00 & 1.74 & 0.27 & 1.09 & 0.22 & -0.67 & 0.78 & 0.62 & 0.67 & -0.48 & 1.39 & 0.86 \\
\hline MIG & 0.31 & 0.38 & -0.26 & -0.18 & -0.94 & -0.07 & -0.04 & 0.10 & -0.18 & -0.17 & -0.20 & 0.25 \\
\hline MIP1A & 0.71 & 0.77 & -0.16 & 0 & -1.04 & 0.13 & 0.10 & -0.15 & 0.17 & 0 & -0.18 & 0.24 \\
\hline MIP1B & 0.44 & 0.82 & -0.17 & -0.04 & -0.77 & -0.20 & 0.08 & -0.10 & -0.10 & 0.14 & -0.06 & 0.42 \\
\hline RANTES & 0.04 & 0.08 & -0.02 & -0.03 & -0.02 & 0.01 & 0.03 & -0.04 & -0.01 & -0.06 & -0.07 & -0.01 \\
\hline TNFA & 0.30 & 0.58 & -0.19 & -0.02 & -1.34 & -0.11 & 0 & -0.32 & 0.11 & -0.21 & -0.12 & 0.15 \\
\hline VEGF & 3.18 & 3.71 & 0.32 & 0.31 & 1.16 & 0.14 & 0.67 & 0.73 & 0.20 & 0.67 & 0.44 & 1.57 \\
\hline IP10 & 0.33 & 0.71 & -0.10 & -0.23 & -1.12 & -0.07 & -0.10 & -0.15 & 0 & -0.17 & -0.03 & 0.13 \\
\hline & $\mathrm{DH} 27$ & JP56 & LB98 & IC70 & IL40 & LJ80 & EM61 & KP48 & KR49 & LB26 & DR61 & JM39 \\
\hline
\end{tabular}

Figure 4. Inflammatory profiles in serum samples from RT-challenged Rhesus macaques. Heatmap presentation of log ${ }_{2}$-fold change values of cytokines, chemokines, and growth factors in the sera of 12 animals, comparing samples collected 24 hours after challenge with those collected 7 days before challenge. Animals are separated by column into their respective groups: control, 4-hour huPB10 intervention, and 12-hour huPB10 intervention. The specific cytokine, chemokine, or growth factor analyzed is indicated on the left. Boxes are color-coded (blue to red) according to scale bar shown on the right. Values are derived from a single 29-plex Luminex analysis.

target $(9,28-31)$. We assume that huPB10, which was readily detected in BALF 12-20 hours following i.v. delivery, exerts its neutralizing effect through the formation of RT-immune complexes in the alveolar space and/or interstitial fluids, thereby blocking toxin uptake by macrophage and epithelial cells, as well as limiting absorption into the circulation $(18,32,33)$.

The other major findings from the current study stem from the profiling of inflammatory markers conducted on Rhesus macaque serum samples and BALF collected before and after RT challenge in both control and huPB10-treated cohorts of animals. First, the inflammatory markers observed in the serum and BAL associated with RT exposure, such as IL-6, IL-1RA, VEGF, MCP1, MIF, and GMCSF, correlate with degrees of edema, PMN infiltration, and overall acute lung injury (34-39). Increased vascular permeability and alveolar epithelial leakage could be attributed to the release of vasoactive amines, such as histamine by tissue mast cells, and cytokines like IL-1 and TNFA chiefly produced by macrophages (40). In fact, there is a significant increase in IL-1B across groups that declined progressively according to the time of huPB10 intervention. The least change in IL-1B was recorded in the 4-hour intervention group, where edema was effectively absent. The relatively modest changes in TNFA levels were surprising and may be due to its short half-life ( $\sim 20$ minutes) relative to the time of sample collection (i.e., 24 hours). Given the prominence of the edema and its likely role in the lethal pathogenesis of ricin toxicosis, further elucidation of the causes 


\begin{tabular}{|c|c|c|c|c|c|c|c|c|c|c|c|}
\hline & Control & & & nterven & & & & 12 & interve & ion & \\
\hline EGF & 0.10 & 0.07 & 0.12 & 0.26 & -0.10 & 0.05 & -0.02 & 0.19 & 0.22 & 0.22 & 0.29 \\
\hline Eotaxin & 2.25 & 0.98 & 2.84 & 1.97 & 1.73 & 1.09 & 3.78 & 2.00 & 3.69 & 2.74 & 4.23 \\
\hline FGF basic & 0.62 & 0.41 & 0.13 & 0.14 & -0.08 & 0.03 & 0.62 & 0.49 & 0.45 & 0.56 & 0.62 \\
\hline GCSF & 1.21 & 0.17 & 0.20 & 0.29 & 0.21 & 0.20 & 0.19 & 0.25 & 1.21 & 0.38 & 3.74 \\
\hline GMCSF & 1.02 & 0.26 & 0.50 & 0.64 & 0.80 & 0.57 & 1.00 & 1.23 & 1.29 & 1.11 & 1.65 \\
\hline HGF & 0.21 & 0.09 & 0 & 0.29 & 0.13 & 0.05 & 0.42 & 0.37 & 0.52 & 0.23 & 1.02 \\
\hline IFNG & -0.17 & 0.09 & 0.13 & 0 & 0.11 & 0 & 0.47 & 0.28 & 0.35 & 0.28 & 0.47 \\
\hline IL1B & 1.76 & 0.84 & 0.57 & 2.16 & 0.82 & 1.29 & 1.04 & 1.32 & 1.52 & 2.76 & 2.36 \\
\hline IL1RA & 2.28 & 0.10 & 0.98 & 2.09 & 1.10 & 1.52 & 2.25 & 2.81 & 1.75 & 3.72 & 3.41 \\
\hline IL2 & 0.92 & 0.03 & 0.21 & 0.40 & 0.16 & 0.16 & 0.90 & 0.90 & 1.04 & 0.88 & 1.39 \\
\hline IL4 & 0.22 & 0.15 & 0.08 & 0.03 & 0.12 & 0 & 0.35 & 0.14 & 0.22 & 0.29 & 0.51 \\
\hline IL5 & 1.07 & 0.25 & 0.58 & 0.63 & 0.28 & 0.29 & 1.12 & 1.56 & 1.46 & 1.43 & 1.90 \\
\hline IL6 & 6.25 & 3.79 & 5.20 & 5.40 & 4.16 & 4.53 & 6.79 & 5.27 & 7.74 & 7.96 & 7.79 \\
\hline IL8 & 0.85 & 0.62 & 0.58 & 0.77 & 1.01 & 0.50 & 0.55 & 1.03 & 1.04 & 1.08 & 1.03 \\
\hline IL10 & 0.08 & 0.04 & 0.08 & 0.06 & 0.03 & 0.01 & 0.27 & 0.73 & 0.34 & 0.23 & 0.53 \\
\hline IL12 & 0.04 & 0.04 & 0.11 & 0.15 & 0.17 & 0.12 & 0.28 & 0.24 & 0.26 & 0.25 & 0.55 \\
\hline IL15 & -0.21 & -0.02 & 0.09 & 0.16 & -0.02 & 0.03 & 0.54 & 0.11 & 0.26 & 0.20 & 0.32 \\
\hline IL17 & 0.20 & 0.07 & 0.17 & 0.58 & 0.16 & 0.03 & 0.74 & 0.69 & 1.52 & 0.22 & 0.69 \\
\hline ITAC & -0.81 & 1.84 & 0.93 & 1.65 & 0.64 & 1.63 & 0.33 & 1.04 & 0.75 & 1.78 & 1.42 \\
\hline MCP1 & -0.89 & 2.69 & 2.02 & 3.02 & 3.78 & 3.51 & 3.83 & 3.71 & 5.19 & 1.97 & 4.65 \\
\hline MDC & -0.06 & 1.73 & 1.62 & 2.01 & 1.34 & 2.70 & 1.30 & 1.51 & 0.56 & 2.72 & 3.62 \\
\hline $\mathrm{MIF}$ & 1.47 & 1.10 & 0.73 & 0.51 & 0.73 & 0.46 & -0.48 & 1.11 & 0.07 & -0.48 & -0.86 \\
\hline MIG & 0 & 0 & -0.13 & 0.06 & 0.06 & 0.13 & 0.14 & -0.21 & 0.16 & 0.29 & 0.20 \\
\hline MIP1A & 0.51 & 0.47 & 0.45 & 1.78 & 1.09 & 0.45 & 0.42 & 0.48 & 2.45 & 0.78 & 0.91 \\
\hline MIP1B & 0.64 & 0.06 & 0.13 & 0.27 & 0.13 & 0.07 & 0.72 & 0.49 & 1.42 & 0.46 & 0.81 \\
\hline RANTES & 0.37 & -0.22 & -0.31 & -0.39 & -0.18 & -0.09 & -0.76 & -0.08 & -0.63 & -0.58 & -0.40 \\
\hline TNFA & 0.49 & 0.28 & 0.36 & 0.77 & 0.51 & 0.47 & 0.65 & 0.75 & 0.74 & 0.77 & 1.37 \\
\hline VEGF & -1.36 & -0.24 & 0.50 & -0.29 & 0.27 & 0.14 & 0.62 & -0.30 & 1.34 & 2.02 & 0.39 \\
\hline IP10 & 0.10 & 0.08 & 0.14 & 0.17 & 0.08 & 0.15 & 0.74 & 0.24 & 0.68 & 0.43 & 0.76 \\
\hline & $\mathrm{DH} 27$ & LB98 & IC70 & IL40 & LJ80 & EM61 & KP48 & KR49 & LB26 & DR61 & JM39 \\
\hline
\end{tabular}

Figure 5. Inflammatory profiles of BALF of RT-challenged Rhesus macaques. Heatmap presentation of log -fold change values of cytokines, chemokines, and growth factors in the BALF of 11 animals, comparing samples collected 24 hours after challenge with those collected 7 days before challenge. Animals are separated by column into their respective groups: control, 4-hour huPB10 intervention, and 12-hour huPB10 intervention. The specific cytokine, chemokine, or growth factor analyzed is indicated on the left. Boxes are color-coded (blue to red) according to scale bar shown on the right. Values are derived from a single 29-plex Luminex analysis. For technical reasons, BALF were only collected from 1 of the 2 control animals at the 24-hour post-RT challenge time point.

that may induce or exacerbate this presentation, such as the role of impairment of tight junction proteins and mast cells, may provide a novel insight to ricin immunopathogenesis $(11,41)$

Second, profiling of BALF using the 29-plex monkey cytokine/chemokine/growth factor Luminex array enabled the unbiased clustering of animals by survival outcome and experimental group. In other words, the composite profile of inflammatory markers in BALF, including IL-2, IL-5, GMCSF, MIF, IP10, IL-12, HGF, RANTES, IL-6, and TNFA, were predictive of whether the animals would survive RT exposure. Identifying an inflammatory "fingerprint" associated with RT exposure has obvious applications in diagnostics, especially in the context of biodefense, where early clinical symptoms following exposure to different toxins and pathogens may, in fact, be indistinguishable (42). As noted above, IL-6 levels were particularly elevated following RT exposure, which is consistent with what has been observed in mice (43). IL-6 is a particularly potent driver of pulmonary inflammation and could very well be a major contributor to RT-induced pathology in conjunction with RT's other properties, including the capacity to induce vascular leak syndrome $(44,45)$. Unfortunately, serum profiling using the Luminex array was not able to discriminate between treatment groups or survivors, presumably because of the localized nature of RT-induced inflammation. Nonetheless, establishing biomarkers and inflammatory processes associated with RT intoxication in NHPs and other experimental models like mice $(6,8,31)$ and swine $(10)$ is essential in the 
JCl insIGHT

RESEARCH ARTICLE

A

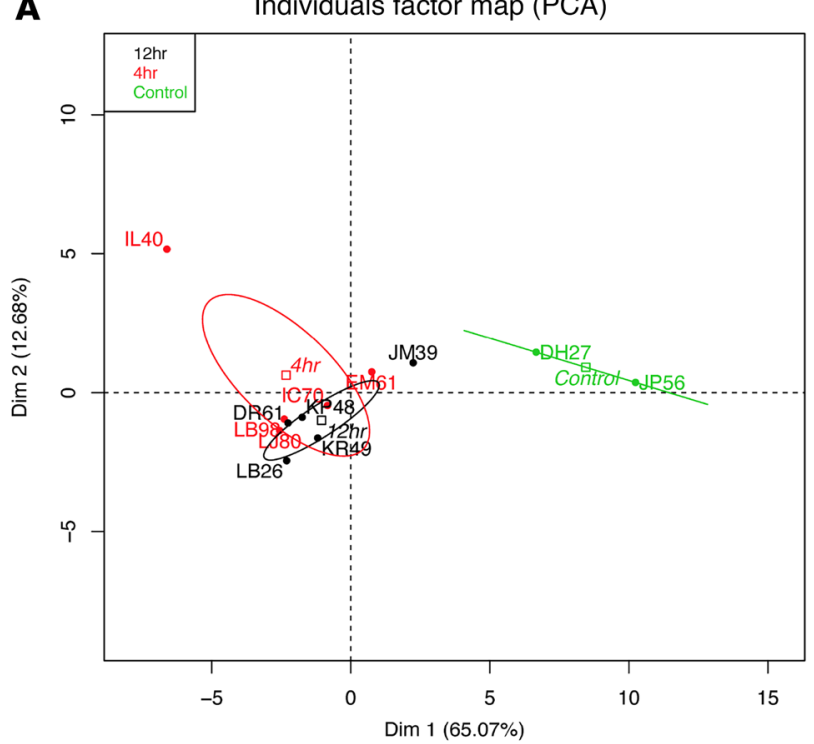

B

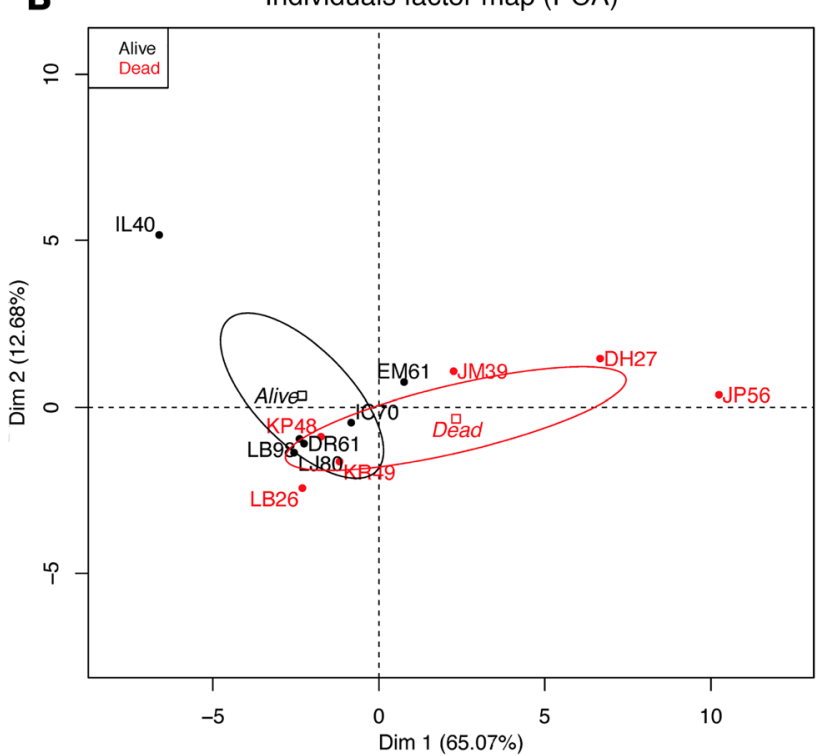

C

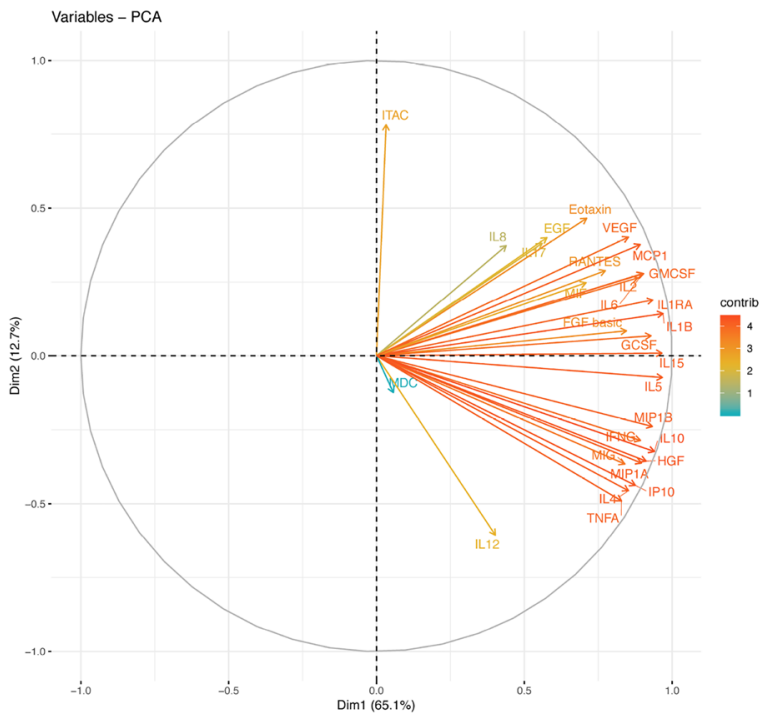

D

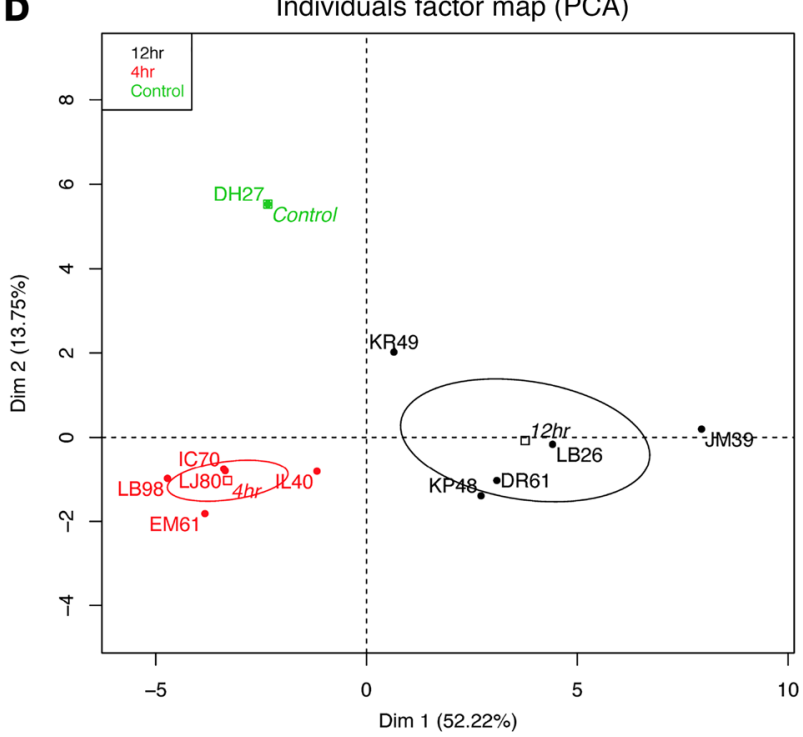

E

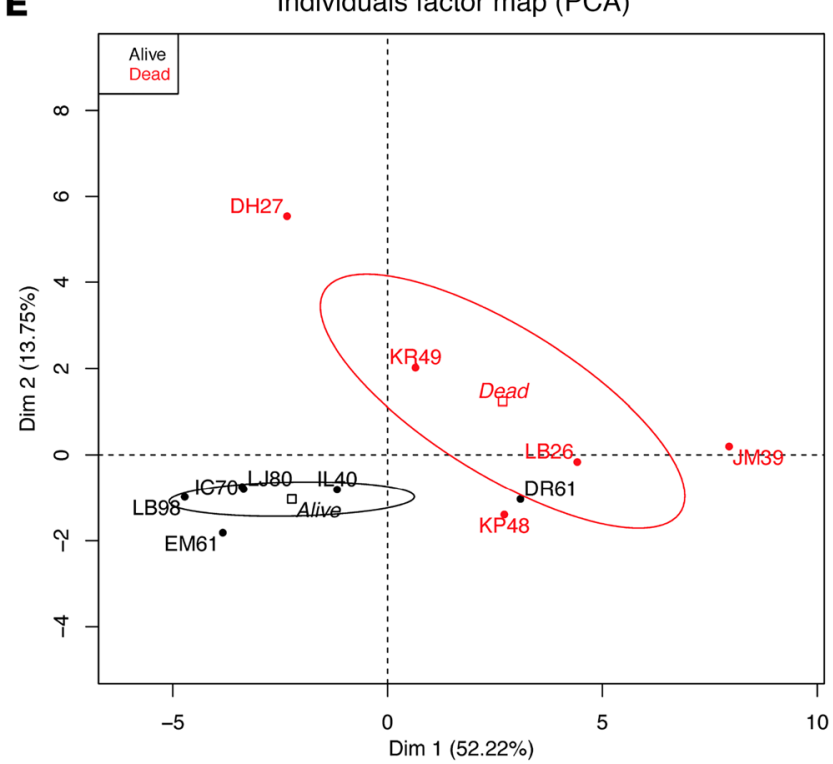

$\mathbf{F}$

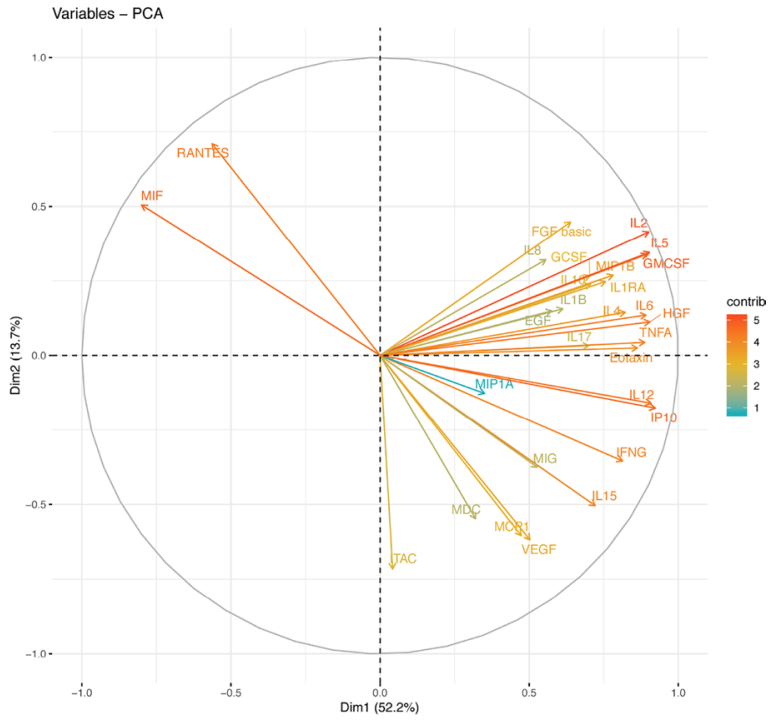

insight.jci.org https://doi.org/10.1172/jci.insight.124771

9 
Figure 6. Principal component analysis of cytokine, chemokine, and growth factor fold changes following RT challenge. Scatter plots of the first 2 principal components of $\log _{2}$-transformed cytokine fold changes in (A-C) serum and (D-F) BAL from all animals included in the study, calculated using singular value decomposition. Each dot represents an individual monkey; red was used for those in the 4-hour group, black for the 12-hour group, and white for the control group. The $95 \%$ confidence ellipses of the group means are color-coded by group. Eigenvectors (C and $\mathbf{F})$ are colored to show the percent contribution of each cytokine to the principal components.

development of a countermeasure for biothreat agents like ricin, since approval of therapeutics for human use must ultimately adhere to the US Food and Drug Administration's (FDA) animal rule.

\section{Methods}

Supplemental Methods are available online with this article

$R T$ and huPB10. Purified RT derived from castor beans (Ricinus communis) was produced as described (46) and used in previous challenge studies with Rhesus macaques (17). huPB10 was expressed using the Nicotiana benthamiana-based manufacturing platform, as described (22). The properties of huPB10 used in this study are shown in Supplemental Table 1.

Animal care and use. Rhesus macaques were born and housed at the TNPRC, which is US Department of Agriculture-licensed and fully accredited by the Association for Assessment and Accreditation of Laboratory Animal Care (AAALAC). Aerosolization, dosing, and delivery of RT were performed as described (17). The $\mathrm{LD}_{50}$ of ricin is $5.8 \mu \mathrm{g} / \mathrm{kg}$ body weight, and the target dose for this experiment was set at the equivalent of $3 \times \mathrm{LD}_{50}$ (approximately $18 \mu \mathrm{g} / \mathrm{kg}$ ). The mean inhaled dose of ricin across all animals was $4.4 \pm 1.4 \mathrm{LD}_{50}$. At 4 hours or 12 hours after exposure, designated animal groups received a single i.v. administration of huPB10 by slow infusion at an individualized unit dose of $10 \mathrm{mg} / \mathrm{kg}$. Sham-treated animals received saline at the 4 hour time point. Treated animals were observed for signs of adverse reactions to the $\mathrm{mAb}$ during administration and throughout the anesthesia recovery period. Animals were bled 7 days before and 24 hours following aerosol challenge. Blood was also collected when the animals either succumbed to intoxication or 14 days after challenge, when the experiment was terminated. BALF were collected from Rhesus macaques 7 days before and 24 hours following aerosol challenge, as described previously (47). Animals that were determined to be in respiratory distress and those that survived for 14 days after exposure to ricin were euthanized by an overdose of sodium pentobarbital, consistent with the recommendation of the American Veterinary Medical Association's Panel on Euthanasia, and submitted for necropsy. After gross necropsy, tissues were collected in neutral buffered zinc-formalin solution (Z-Fix Concentrate, Anatach). Tissues were processed, sectioned, and stained as previously described (4).

$I H C$. Tissue sections, 3-4 $\mu \mathrm{m}$ in thickness, were deparaffinized in CitriSolve (Decon Labs) and rehydrated by processing them through graded alcohols. Antigen unmasking was accomplished by digesting tissue in 10 $\mu \mathrm{g} / \mathrm{ml}$ of protein kinase for 15 minutes at room temperature (Thermo Fisher Scientific). Endogenous enzymes and nonspecific background were blocked with Background Punisher (Biocare Medical), followed by BLOXALL (Vector Laboratories). The primary antibody (clone BMK-13; catalog NBP1-42140; Novus Biologicals) was incubated on the tissue sections at a dilution of 1:100 for 1 hour at room temperature. Subsequently, sections were sequentially incubated with an alkaline phosphatase-based detection polymer kit (MACH 4; Biocare Medical) and Warp Red (Biocare Medical). Sections were counterstained with Tacha's hematoxylin (Biocare Medical) and mounted using a permanent mounting medium (EcoMount; Biocare Medical).

Statistics. Statistical analysis was carried out with GraphPad Prism 6 (GraphPad Software). The difference in outcomes between groups was determined by Fisher's exact test (2-tailed), and the mean survival times after exposure to RT were compared by log-rank analysis of Kaplan-Meier survival curves. The statistical significance of the effects of ricin challenge and huPB10 intervention on cytokine/chemokine/ growth factor levels were analyzed with 2-way repeated-measures ANOVA in both serum and BAL, with the repeated measures being before and after exposure status and with treatment group as the independent measure. Resulting $P$ values were corrected with the Benjamini, Krieger, and Yekutieli method to control FDR. Due to the low abundance of many of the inflammatory markers in serum and BALF examined by Luminex, all analyses were performed on $\log _{2}$-transformed raw fluorescent intensity values to avoid the need to censor values (48). A $P$ value less than 0.05 was considered significant.

PCA was performed using singular value decomposition with the R package FactoMineR (49). Heatmap construction using raw fold-change values was done using GraphPad Prism 6. Hierarchical clustering and scaled heatmap construction were completed with the R package pheatmap (50). 
Study approval. The studies described involving Rhesus macaques were approved as protocol P0334 by the IACUC at Tulane University. Animal studies were conducted in strict compliance with protocols approved by TNPRC's IACUC.

\section{Author contributions}

NB, OB, DK, MP, KW, and ESV generated reagents; PJD, LDM, YR, FJTV, and CJR conducted animal studies and animal tissue/sample analysis; DJE performed statistical analysis; ESV provided critical reagents; CJR, NJM, and LZ are responsible for experimental design. CJR, LZ, and NM prepared and edited the manuscript.

\section{Acknowledgments}

We thank the Wadsworth Center's Biochemistry and Immunology core facility for assistance with flow cytometry and the Histopathology Core facility for assistance with tissue section preparations. This work was supported by R01AI098774 to LZ and contract no. HHSN272201400021C and R01 AI125190 to NJM from the National Institutes of Allergy and Infectious Diseases, NIH. The work at TNPRC was also supported by grant OD011104 to CJR from the National Center for Research Resources and the Office of Research Infrastructure Programs (ORIP), NIH. The content is solely the responsibility of the authors and does not necessarily represent the official views of the NIH. The funders had no role in study design, data collection and analysis, decision to publish, or preparation of the manuscript.

Address correspondence to: Chad J. Roy, Tulane National Primate Research Center, Microbiology Division, 18703 Three Rivers Road, Covington, Louisiana 70433, USA. Phone: 985.871.6417; Email: croy@ tulane.edu. Or to: Larry Zeitlin, Mapp Biopharmaceutical Inc., 6160 Lusk Boulevard, San Diego, California 92121, USA. Phone: 858.625.0335; Email: larry.zeitlin@mappbio.com. Or to: Nicholas Mantis, Division of Infectious Disease, Wadsworth Center, 120 New Scotland Avenue, Albany, New York 12208, USA. Phone: 518.473.7487; Email: nicholas.mantis@health.ny.gov.

1. Cieslak TJ, et al. Beyond the Dirty Dozen: A Proposed Methodology for Assessing Future Bioweapon Threats. Mil Med. 2018;183(1-2):e59-e65.

2. Wolfe DN, Florence W, Bryant P. Current biodefense vaccine programs and challenges. Hum Vaccin Immunother. 2013;9(7):1591-1597.

3. Gal Y, Mazor O, Falach R, Sapoznikov A, Kronman C, Sabo T. Treatments for Pulmonary Ricin Intoxication: Current Aspects and Future Prospects. Toxins (Basel). 2017;9(10):E311.

4. Pincus SH, Bhaskaran M, Brey RN, Didier PJ, Doyle-Meyers LA, Roy CJ. Clinical and Pathological Findings Associated with Aerosol Exposure of Macaques to Ricin Toxin. Toxins (Basel). 2015;7(6):2121-2133.

5. Wilhelmsen CL, Pitt ML. Lesions of acute inhaled lethal ricin intoxication in rhesus monkeys. Vet Pathol. 1996;33(3):296-302.

6. Poli MA, Rivera VR, Pitt ML, Vogel P. Aerosolized specific antibody protects mice from lung injury associated with aerosolized ricin exposure. Toxicon. 1996;34(9):1037-1044.

7. Pratt TS, Pincus SH, Hale ML, Moreira AL, Roy CJ, Tchou-Wong KM. Oropharyngeal aspiration of ricin as a lung challenge model for evaluation of the therapeutic index of antibodies against ricin A-chain for post-exposure treatment. Exp Lung Res. 2007;33(8-9):459-481.

8. Wong J, Korcheva V, Jacoby DB, Magun B. Intrapulmonary delivery of ricin at high dosage triggers a systemic inflammatory response and glomerular damage. Am J Pathol. 2007;170(5):1497-1510.

9. Lindauer ML, Wong J, Iwakura Y, Magun BE. Pulmonary inflammation triggered by ricin toxin requires macrophages and IL-1 signaling. J Immunol. 2009;183(2):1419-1426.

10. Katalan S, et al. A novel swine model of ricin-induced acute respiratory distress syndrome. Dis Model Mech. 2017;10(2):173-183

11. Sapoznikov A, et al. Early disruption of the alveolar-capillary barrier in a ricin-induced ARDS mouse model: neutrophil-dependent and -independent impairment of junction proteins [published online ahead of print November 1, 2018]. Am J Physiol Lung Cell Mol Physiol. https://doi.org/10.1152/ajplung.00300.2018.

12. Spooner RA, Lord JM. How ricin and Shiga toxin reach the cytosol of target cells: retrotranslocation from the endoplasmic reticulum. Curr Top Microbiol Immunol. 2012;357:19-40.

13. Endo Y, Mitsui K, Motizuki M, Tsurugi K. The mechanism of action of ricin and related toxic lectins on eukaryotic ribosomes. The site and the characteristics of the modification in $28 \mathrm{~S}$ ribosomal RNA caused by the toxins. $J$ Biol Chem. 1987;262(12):5908-5912.

14. Endo Y, Tsurugi K. RNA N-glycosidase activity of ricin A-chain. Mechanism of action of the toxic lectin ricin on eukaryotic ribosomes. J Biol Chem. 1987;262(17):8128-8130.

15. Tesh VL. The induction of apoptosis by Shiga toxins and ricin. Curr Top Microbiol Immunol. 2012;357:137-178.

16. Brown RF, White DE. Ultrastructure of rat lung following inhalation of ricin aerosol. Int J Exp Pathol. 1997;78(4):267-276.

17. Roy CJ, et al. Thermostable ricin vaccine protects rhesus macaques against aerosolized ricin: Epitope-specific neutralizing antibodies correlate with protection. Proc Natl Acad Sci USA. 2015;112(12):3782-3787.

18. O'Hara JM, Neal LM, McCarthy EA, Kasten-Jolly JA, Brey RN, Mantis NJ. Folding domains within the ricin toxin A subunit 
as targets of protective antibodies. Vaccine. 2010;28(43):7035-7046.

19. Toth RT, et al. High-Definition Mapping of Four Spatially Distinct Neutralizing Epitope Clusters on RiVax, a Candidate Ricin Toxin Subunit Vaccine. Clin Vaccine Immunol. 2017;24(12):e00237-17.

20. Vance DJ, Mantis NJ. Resolution of two overlapping neutralizing B cell epitopes within a solvent exposed, immunodominant $\alpha$-helix in ricin toxin's enzymatic subunit. Toxicon. 2012;60(5):874-877.

21. Sully EK, et al. A tripartite cocktail of chimeric monoclonal antibodies passively protects mice against ricin, staphylococcal enterotoxin B and Clostridium perfringens epsilon toxin. Toxicon. 2014;92:36-41.

22. Van Slyke G, et al. Humanized Monoclonal Antibody That Passively Protects Mice against Systemic and Intranasal Ricin Toxin Challenge. Clin Vaccine Immunol. 2016;23(9):795-799.

23. Bhaskaran M, Didier PJ, Sivasubramani SK, Doyle LA, Holley J, Roy CJ. Pathology of lethal and sublethal doses of aerosolized ricin in rhesus macaques. Toxicol Pathol. 2014;42(3):573-581.

24. Reisler RB, Smith LA. The need for continued development of ricin countermeasures. Adv Prev Med. 2012;2012:149737.

25. Vance DJ, Mantis NJ. Progress and challenges associated with the development of ricin toxin subunit vaccines. Expert Rev Vaccines. 2016;15(9):1213-1222

26. Roy CJ, Song K, Sivasubramani SK, Gardner DJ, Pincus SH. Animal models of ricin toxicosis. Curr Top Microbiol Immunol. 2012;357:243-257.

27. Sully EK, et al. Chimeric plantibody passively protects mice against aerosolized ricin challenge. Clin Vaccine Immunol. 2014;21(5):777-782.

28. Cook DL, David J, Griffiths GD. Retrospective identification of ricin in animal tissues following administration by pulmonary and oral routes. Toxicology. 2006;223(1-2):61-70.

29. Doebler JA, et al. The distribution of [125I]ricin in mice following aerosol inhalation exposure. Toxicology. 1995;98(1-3):137-149.

30. Falach R, et al. Quantitative profiling of the in vivo enzymatic activity of ricin reveals disparate depurination of different pulmonary cell types. Toxicol Lett. 2016;258:11-19.

31. Wong J, Korcheva V, Jacoby DB, Magun BE. Proinflammatory responses of human airway cells to ricin involve stress-activated protein kinases and NF-kappaB. Am J Physiol Lung Cell Mol Physiol. 2007;293(6):L1385-L1394.

32. O'Hara JM, Mantis NJ. Neutralizing monoclonal antibodies against ricin's enzymatic subunit interfere with protein disulfide isomerase-mediated reduction of ricin holotoxin in vitro. J Immunol Methods. 2013;395(1-2):71-78.

33. Yermakova A, Klokk TI, O'Hara JM, Cole R, Sandvig K, Mantis NJ. Neutralizing Monoclonal Antibodies against Disparate Epitopes on Ricin Toxin's Enzymatic Subunit Interfere with Intracellular Toxin Transport. Sci Rep. 2016;6:22721.

34. Kaner RJ, Ladetto JV, Singh R, Fukuda N, Matthay MA, Crystal RG. Lung overexpression of the vascular endothelial growth factor gene induces pulmonary edema. Am J Respir Cell Mol Biol. 2000;22(6):657-664.

35. Guo RF, et al. Regulatory effects of eotaxin on acute lung inflammatory injury. J Immunol. 2001;166(8):5208-5218.

36. Marsh LM, et al. Surface expression of CD74 by type II alveolar epithelial cells: a potential mechanism for macrophage migration inhibitory factor-induced epithelial repair. Am J Physiol Lung Cell Mol Physiol. 2009;296(3):L442-L452.

37. Sauler M, et al. Macrophage migration inhibitory factor deficiency in chronic obstructive pulmonary disease. Am J Physiol Lung Cell Mol Physiol. 2014;306(6):L487-L496.

38. Sauler M, et al. Endothelial CD74 mediates macrophage migration inhibitory factor protection in hyperoxic lung injury. FASEB J. 2015;29(5):1940-1949.

39. Ward PA. Role of complement, chemokines, and regulatory cytokines in acute lung injury. Ann N Y Acad Sci. 1996;796:104-112.

40. Wong J, Magun BE, Wood LJ. Lung inflammation caused by inhaled toxicants: a review. Int J Chron Obstruct Pulmon Dis. 2016;11:1391-1401

41. Virk H, Arthur G, Bradding P. Mast cells and their activation in lung disease. Transl Res. 2016;174:60-76.

42. Lipscomb MF, Hutt J, Lovchik J, Wu T, Lyons CR. The pathogenesis of acute pulmonary viral and bacterial infections: investigations in animal models. Annu Rev Pathol. 2010;5:223-252.

43. Sabo T, et al. Antibody treatment against pulmonary exposure to abrin confers significantly higher levels of protection than treatment against ricin intoxication. Toxicol Lett. 2015;237(2):72-78.

44. Rubini A. Interleukin-6 and lung inflammation: evidence for a causative role in inducing respiratory system resistance increments. Inflamm Allergy Drug Targets. 2013;12(5):315-321.

45. Soler-Rodríguez AM, Ghetie MA, Oppenheimer-Marks N, Uhr JW, Vitetta ES. Ricin A-chain and ricin A-chain immunotoxins rapidly damage human endothelial cells: implications for vascular leak syndrome. Exp Cell Res. 1993;206(2):227-234.

46. Ghetie MA, Ghetie V, Vitetta ES. Immunotoxins for the treatment of B-cell lymphomas. Mol Med. 1997;3(7):420-427.

47. Tate MK, Rico PJ, Roy CJ. Comparative study of lung cytologic features in normal rhesus (Macaca mulatta), cynomolgus (Macaca fasicularis), and African green (Chlorocebus aethiops) nonhuman primates by use of bronchoscopy. Comp Med. 2004;54(4):393-396.

48. Breen EJ, Tan W, Khan A. The Statistical Value of Raw Fluorescence Signal in Luminex xMAP Based Multiplex Immunoassays. Sci Rep. 2016;6:26996.

49. Lê S, Josse J, Husson F. FactoMineR: An R Package for Multivariate Analysis. Journal of Statistical Software. 2008;25(1):1-18.

50. Kolde R. pheatmap: Pretty Heatmaps. R Project. https://cran.r-project.org/web/packages/pheatmap/index.html. Updated May 19, 2018. Accessed December 11, 2018. 